\title{
HOLONOMY, RICCI TENSOR AND KILLING VECTOR FIELDS ${ }^{1}$
}

\section{KATSUMI NOMIZU}

1. Let $M$ be a Riemannian manifold, connected and of class $C^{\infty}$. For any vector field $X$ on $M$, we define a tensor field $A_{X}$ of type $(1,1)$, namely, a field of linear endomorphisms of the tangent space at each point, by setting $A_{X} \cdot Y=-\nabla_{Y} X$, where $Y$ is a tangent vector at an arbitrary point and $\nabla_{Y}$ denotes covariant derivative with respect to $Y$.

It is known [2] that if $X$ is a Killing vector field, then $A_{X}$ is a skew-symmetric endomorphism of the tangent space and belongs to the normalizer of the holonomy algebra (Lie algebra of the homogeneous holonomy group) at each point of $M$. A result of Lichnerowicz [3] implies that if the restricted homogeneous holonomy group is irreducible and if the Ricci tensor is not zero, then $A_{X}$ belongs to the holonomy algebra at each point. One of the basic contributions in contemporary Riemannian geometry is the result, due to Kostant [2], that the same conclusion holds if $M$ is compact. His proof uses the Green-Stokes formula which is valid only on a compact Riemannian manifold.

In the present note, we wish to provide a more geometric proof to this theorem of Kostant, in fact, in a generalized form where the compactness of the space is not assumed. Namely, we shall prove

Theorem. Let $M$ be a complete Riemannian manifold. If $X$ is a Killing vector field defined on $M$ which attains a local maximum in its length at some point of $M$, then $A_{X}$ belongs to the holonomy algebra at each point of $M$.

Here we say that the length $\|X\|$ of a vector field $X$ attains a local maximum at a point $x \in M$ if $x$ has a neighborhood $U$ such that $\|X\|_{y} \leqq\|X\|_{x}$ for every point $y \in U$. The assumption of our theorem is valid, for example, if $X$ has constant length on $M$, or if $M$ is compact. The theorem of Kostant follows immediately.

2. We now sketch the proof of our theorem. First, we may assume that $M$ is simply connected. Otherwise, let $\hat{M}$ be the universal covering manifold of $M$ provided with a natural Riemannian metric so

\footnotetext{
Presented to the Society, October 22, 1960; received by the editors September 9, 1960.

1 This work was done while the author received Grant No. 8206 from the National Science Foundation at the Catholic University of America.
} 
that the projection $\pi$ of $\hat{M}$ onto $M$ is a local isometry. $\hat{M}$ is complete. The holonomy algebra at $\hat{x} \in \hat{M}$ is the same as the holonomy algebra at $x=\pi(\hat{x})$. The given Killing vector field $X$ on $M$ can be lifted to a Killing vector field $\hat{X}$ on $\hat{M}$ which projects upon $X$. Suppose that $\|X\|$ attains a local maximum at $x \in M$. It is then clear that $\|\hat{X}\|$ attains a local maximum at any point $\hat{x} \in \hat{M}$ such that $\pi(\hat{x})=x$. If the conclusion of the theorem holds for $A_{\widehat{X}}$, then it holds obviously for $A_{X}$.

Thus, let us assume that $M$ is simply connected and complete. Let $M=M_{0} \times M_{1} \times \cdots \times M_{k}$ be the de Rham decomposition of $M$, where $M_{0}$ is a euclidean space and each $M_{i}, 1 \leqq i \leqq k$, is irreducible [4]. The given Killing vector field $X$ on $M$ decomposes into a sum $X_{0}+X_{1}+\cdots+X_{k}$, where each $X_{i}, 0 \leqq i \leqq k$, is a Killing vector field on $M_{i}$. Let $x=\left(x_{0}, x_{1}, \cdots, x_{k}\right) \in M_{0} \times M_{1} \times \cdots \times M_{k}$ be a point where $\|X\|$ attains a local maximum. Since $\|X\|_{x}^{2}=\left\|X_{0}\right\|_{x_{0}}^{2}$ $+\left\|X_{1}\right\|_{x_{1}}^{2}+\cdots+\left\|X_{k}\right\|_{x_{k}}^{2}$, each vector field $X_{i}$ on $M_{i}$ attains a local maximum at the point $x_{i}$ for the following reason. Suppose that this is not the case and that for some $i, x_{i} \in M_{i}$ has an arbitrarily nearby point $y_{i} \in M_{i}$ such that $\left\|X_{i}\right\|_{y_{i}}>\left\|X_{i}\right\|_{x_{i}}$. Then we can get a point $y=\left(x_{0}, x_{1}, \cdots, y_{i}, \cdots, x_{k}\right)$ at which $\|X\|_{y}>\|X\|_{x}$ and which is arbitrarily near $x$, contrary to the assumption that $\|X\|$ attains a local maximum at $x$. It is clear that we have only to prove the theorem for each $X_{i}$ on $M_{i}$.

We now consider each vector field $X_{i}$ on $M_{i}$. On the euclidean space $M_{0}$, the length of a Killing vector field $X_{0}$ cannot have a local maximum unless it is constant, in which case, $X_{0}$ is a parallel vector field and the corresponding endomorphism $A_{X_{0}}$ is zero at every point. On each $M_{i}, 1 \leqq i \leqq k$, we make the following argument. The holonomy algebra of $M_{i}$ is irreducible. If the Ricci tensor of $M_{i}$ is not identically zero, then $A_{X_{i}}$ belongs to the holonomy algebra by the result of Lichnerowicz as we already mentioned. To deal with the factor $M_{i}$ whose Ricci tensor is identically zero, we need the following two general lemmas whose proofs will be given in $\$ 4$.

Leмma 1. Let $X$ be a Killing vector field on a Riemannian manifold. Then

$$
\operatorname{div}\left(A_{X} \cdot X\right)=-S(X, X)-\operatorname{trace}\left(A_{X}^{2}\right)
$$

where $S(X, X)$ is the quadratic form in $X$ given by the Ricci tensor.

LEMMA 2. For a Killing vector field $X$ on a Riemannian manifold with Riemannian metric $g$, let $\phi=(1 / 2)\|X\|^{2}$. For any vector field $V$ with $\nabla_{V} V=0$ in a neighborhood of a point $x$, we have 


$$
V^{2} \phi=g\left(V, \nabla_{V}\left(A_{X} \cdot X\right)\right) .
$$

Now assume that the Ricci tensor $S$ is identically zero. Lemma 1 gives $\operatorname{div}\left(A_{X} \cdot X\right)=-\operatorname{trace}\left(A_{X}\right)^{2}$. If $\|X\|$ attains a local maximum at $x$, we have $V^{2} \phi=g\left(V, \nabla_{V}\left(A_{X} \cdot X\right)\right) \leqq 0$ at $x$ in Lemma 2. Since $\operatorname{div}\left(A_{X} \cdot X\right)$ is the trace of the linear mapping $V \rightarrow \nabla_{V}\left(A_{X} \cdot X\right)$ of the tangent space at $x$ into itself, we see that $\operatorname{div}\left(A_{X} \cdot X\right) \leqq 0$ at $x$. On the other hand, $A_{X}$ being skew-symmetric, we have $\operatorname{trace}\left(A_{X}\right)^{2} \leqq 0$ at $x$. Therefore we must have $\operatorname{div}\left(A_{X} \cdot X\right)=\operatorname{trace}\left(A_{X}\right)^{2}=0$ at $x$, which is possible only when $A_{X}=0$ at $x$. Thus $A_{X}$ belongs, of course, to the holonomy algebra at $x$. As was shown in [2], it follows that $A_{X}$ belongs to the holonomy algebra at each point of $M$. This concludes the proof of our theorem.

3. A similar argument allows us to prove a theorem of Bochner [1] in the following form.

Theorem. Let $M$ be a Riemannian manifold whose Ricci tensor is negative definite. If a Killing vector field $X$ attains a local maximum in its length at some point of $M$, then $X$ is identically zero.

In fact, by the same argument following the above lemmas, we have $-S(X, X)-\operatorname{trace}\left(A_{X}\right)^{2} \leqq 0$ at $x$. On the other hand, since $S$ is negative definite, we must have $-S(X, X) \geqq 0$ everywhere. We have also $-\operatorname{trace}\left(A_{X}\right)^{2} \geqq 0$. Thus, at $x$, we have $S(X, X)=0$ and $\operatorname{trace}\left(A_{X}\right)^{2}$ $=0$, which imply that $X=0$ and $A_{X}=0$ at $x$. By a well known fact that a Killing vector field on a connected Riemannian manifold is uniquely determined by the values of $X$ and $A_{X}$ at an arbitrary single point [2], we see that $X$ is zero on the whole manifold.

4. For the sake of completeness, we shall give here proofs of Lemmas 1 and $2 .^{2}$

Proof of Lemma 1. The Ricci tensor is given, by definition, by $S(X, Y)=$ trace of the linear endomorphism $V \rightarrow R(V, X) Y$ of the tangent space at each point, where $R$ is the curvature tensor and $R(V, Y)$ is the skew-symmetric endomorphism obtained by contraction of $R$ with vectors $V$ and $Y$. Now assume that $X$ is a Killing vector field and $Y$ is an arbitrary vector field. We have $\nabla_{V}\left(A_{X}\right)=R(X, V)$ (see [2]), and hence $-R(X, V) Y=-\left(\nabla_{V}\left(A_{X}\right)\right) Y=-\nabla_{V}\left(A_{X} \cdot Y\right)$ $+A_{X}\left(\nabla_{V} Y\right)=-\nabla_{V}\left(A_{X} \cdot Y\right)-A_{X} \cdot A_{Y} \cdot V$. Thus we obtain

$$
S(X, Y)=-\operatorname{div}\left(A_{X} \cdot Y\right)-\operatorname{trace}\left(A_{X} A_{Y}\right)
$$

${ }^{2}$ After the completion of this paper, there appeared a paper by Robert Hermann, Totally geodesic orbits of groups of isometries (Lincoln Laboratory, MIT, June 1960). He makes use of formulas which are essentially the same as ours. 
The formula in Lemma 1 follows by taking $Y=X$.

Proof of Lemma 2. We recall that $Z \cdot g(X, Y)=g\left(\nabla_{z} X, Y\right)$ $+g\left(X, \nabla_{Z} Y\right)$ for arbitrary vector fields $X, Y$ and $Z$ (this is an infinitesimal expression of the fact that the parallel displacement of the Riemannian connection is isometric). Applying this formula, we have $V \cdot \phi=(1 / 2) V \cdot g(X, X)=g\left(\nabla_{V} X, X\right)=g\left(-A_{X} \cdot V, X\right)=g\left(V, A_{X} \cdot X\right)$, since $A_{X}$ is skew-symmetric when $X$ is a Killing vector field. We then obtain

$V^{2} \phi=V \cdot g\left(V, A_{X} X\right)=g\left(\nabla_{V} V, A_{X} \cdot X\right)+g\left(V, \nabla_{V}\left(A_{X}\right)\right)=g\left(V, \nabla_{V}\left(A_{X}\right)\right)$, since $\nabla_{V} V=0$ by assumption.

\section{BIBLIOGRAPHY}

1. S. Bochner, Vector fields and Ricci curvature, Bull. Amer. Math. Soc. vol. 52 (1946) pp. 776-797.

2. B. Kostant, Holonomy and the Lie algebra of infinitesimal motions of a Riemannian manifold, Trans. Amer. Math. Soc. vol. 80 (1955) pp. 528-542.

3. A. Lichnerowicz, Espaces homogènes kähleriens, Colloque de Géométrie Différentielle, Strasbourg, 1953, pp. 171-184.

4. G. de Rham, Sur la réductibilité d'un espace de Riemann, Comment. Math. Helv. vol. 26 (1952) pp. 328-344.

BROWN UNIVERSITY AND

Catholic University of America 